\title{
Compatible solutes in halophilic filamentous fungi
}

\author{
Sarita W. Nazareth ${ }^{1}$, Valerie Gonsalves ${ }^{1,2} *$ and Sushama M. Gaikwad ${ }^{3}$ \\ ${ }^{\prime}$ Department of Microbiology, Goa University, Taleigao Plateau, Goa 403206, India. \\ ${ }^{2}$ Department of Microbiology, St. Xavier's College, Mapusa, Goa \\ ${ }^{3}$ Division of Biochemical Sciences, National Chemical Laboratory, Dr. HomiBhabha Road, Pune 411008, India. \\ *Corresponding author Email: valerie.gonsalves@gmail.com
}

(Submitted on August 18, 2019; Accepted on November 22, 2019)

\section{ABSTRACT}

\begin{abstract}
Halophilic fungi combat the osmotic stress of their saline environment by the accumulation of compatible solutes, also termed as osmolytes. Filamentous fungi isolated from various athalassohaline, thalassohaline and polyhaline econiches were selected from different genera and species, on the basis of their classification as obligate and facultative halophiles. The salt tolerance index of the isolates proved their euryhaline nature, able to adapt to a wide range of salt concentrations, the obligate halophiles however, showing a tendency to a stenohaline nature, with a narrowertolerance range. Examination of the osmolyte production indicated that sucrose, trehalose, arabitol, erythritol, inositol, mannitol, dulcitol, xylitol, galactose and glucose were present, the polyols being found in each of the isolates studied. The variations in concentrations of osmolyte pools vis-à-vis the salt tolerance characteristics in the different genera and species, did not indicate any correlation with the obligate or facultative halophilic nature of the fungi, or with the saline econiches from which they were obtained, as well as with the different genera. However, the similarity in the different types of osmolytes between the different genera and species, indicated their notable role in osmoadaptation in fungi in general.
\end{abstract}

KEYWORDS: Osmolyte, halophilic, fungi, hypersaline, polyhaline

\section{INTRODUCTION}

Halophilic microorganisms are exposed to a high osmotic pressure of hypertonic saline environments and develop strategies of osmoregulation to maintain iso-osmotic balance with their surroundings, to enable growth and survival in the otherwise harsh conditions of hypersalinity and the resultant low water activity of their environment (Kunte et al., 2002; Grant, 2004). While in archaea, osmoadaptation occurs mainly by a salt-in-cytoplasm mechanism, eubacteria and fungi survive by the build-up of compatible solutes or osmolytes, so termed because these do not interfere with the metabolic processes of the organism (DasSarma and Arora, 2002).

Halophilic fungi have been found to either have obligate requirement of sodium chloride for its growth, or are able to grow even without $\mathrm{NaCl}$, and have been termed as obligate or true halophiles, and as facultative halophiles, respectively (Nazareth et al., 2012). Reports on osmoadaptation in fungi and/or their survival in hypersaline environments, have focused on the synthesis of melanin in black yeasts and dematiaceous fungi (Kogej et al., 2007), mycosporins in black yeasts, ascomycetes and basidiomycetes (Kogej et al., 2006) and some compatible solutes such as glycerol, erythritol, arabitol, ribitol and mannitol, trehalose and glucose, in black yeasts and mycorrhizal fungi, as well as in a few filamentous fungi (Beever and Laracy, 1986; Kelavkar and Chhatpar, 1993; El-Kady et al., 1995; Nesci et al., 2004; Ramirez et al., 2004; Roberts, 2005; Bois et al., 2006; Kogej et al., 2007). These fungi had been grown in the presence of salt but the halophilic nature was not determined (Nazareth, 2014). Moreover, only a single or a few select organisms have been studied from a specific sample.

This paper presents the types of osmolytes produced by halophilic fungi, and an analysis on the correlation of types of osmolytes accumulated in both obligate and facultative halophiles, and/or the habitat from which they have been isolated, namely, athalassohaline and thalassohaline, hypersaline and polyhaline environments. An examination is also made with respect to osmolyte production by the different genera.

\section{MATERIALSAND METHODS}

Isolates: Thirteen halophilic fungi were selected, previously isolated from different saline environments: the Dead Sea water (DSw) and sediment (DSs) samples, with a salinity of $370 \%$ and $450 \%$, respectively (Nazareth et al., 2012), from the estuary of Mandovi, Goa, on the West Coast of the Indian peninsula-from surface and bottom waters $\left(\mathrm{EMw}_{\mathrm{s}}\right.$ and $\left.\mathrm{EMw}_{\mathrm{b}}\right)$ and from sediment (EMs) samples, with a salinity range of $13 \%$ - $31 \%$ from upstream travelling towards the mouth, with the salinity of the sediment being 5\%o-10\% (Gonsalves et al., 2012), from water samples of mangroves at Ribander (MRw) and sediment of solar salterns, Ribander, Goa (SRs), with salinity of 32\%o and 295\%, respectively (Nayak et al., 2012). The fungi comprised the genera of Aspergillus, Penicillium and Cladosporium, of which some were obligate halophiles [A. penicillioides DSs40 (GenBank Accession number HQ702385), EM6s137 (JQ240645), EM8 $\mathrm{w}_{\mathrm{s}} 146$ and MRw207] and others were facultative halophiles [A. restrictus DSw14 (HQ702382), A. penicillioides $\mathrm{EM} 7 \mathrm{w}_{\mathrm{s}} 138$, A. versicolor SRs246, A. wentii SRs249, P. corylophilum DSw10, P. waksmanii DSw16, P. corylophilum EM5s132, C. cladosporioides DSw26 and C. carpophilum EM9 $\left.\mathrm{w}_{\mathrm{b}} 154\right]$ which were classified on the basis of their salt requirement for growth, as recorded earlier (Gonsalves et al., 2012; Nayak et al., 2012; Nazareth et al., 2012).

Salt tolerance index: Salt tolerance index of the isolates was obtained from the halotolerance curves performed in triplicate on Czapek Dox Agar (CzA) at $30^{\circ} \mathrm{C}$ (Gonsalves et al., 2012; Nayak et al., 2012; Nazareth et al., 2012) and was calculated as the ratio of growth in terms of colony diameter obtained at different concentrations of solar salt, to the maximum growth obtained at the optimal salt concentration of $5 \%$ or $10 \%$, after $7 \mathrm{~d}$ incubation, as described earlier 
(Nazareth and Gonsalves, 2014a).

Osmolyte analysis: The isolates were grown in Czapek Dox Broth supplemented with a salt concentration that supported maximal growth $(10 \%$ salt for obligate halophiles and $5 \%$ or $10 \%$ salt for facultative halophiles). The cultures were harvested in the logarithmic growth phase, washed with deionised water and osmolytes were extracted by the method of Ramirez et al., (2004). The fungal biomass $(0.5 \mathrm{~g})$ was frozen, then homogenised in $1.5 \mathrm{~mL}$ deionised water, using a mortar and pestle, and sonicated with a B-Braun Labsonic sonicator, at $150 \mathrm{mv}, 10$ pulses for $20 \mathrm{~s}$ with intervals of $10 \mathrm{~s}$ for $5 \mathrm{~min}$, followed by heating for $5.5 \mathrm{~min}$ in a boiling water bath, and cooled. Samples were then centrifuged at 14,000 $\mathrm{rpm}$ for $15 \mathrm{~min}$. The supernatant was filtered through a 0.22 $\mu \mathrm{m}$ Millipore filter and analyzed by HPLC (Waters) using a Sugar Pak column maintained at $70^{\circ} \mathrm{C}$ and refractive index detector; elution was carried out with calcium di-sodium $\operatorname{EDTA}(0.1 \mathrm{mM})$ at a flow rate of $0.4 \mathrm{~mL} \mathrm{~min}^{-1}$. Data analysis of retention time of standards and samples was done using Millenium software. The peaks were compared with calibration curves of standard sugars galactose, glucose, sucrose, trehalose and sugar alcohols arabitol, dulcitol, erythritol, glycerol, inositol, mannitol, ribitol, sorbitol and xylitol $\left(2 \mathrm{mg} \mathrm{mL}^{-1}\right)$. Sugar and polyol concentrations of the samples were calculated as $\mathrm{mg} \mathrm{g}^{-1}$ wet weight of mycelia.

\section{RESULTS}

Salt tolerance index: The salt tolerance indices (STI) of the isolates at different solar salt concentrations, given in Fig. 1, indicated that the obligate halophilic isolates of Aspergillus penicillioides DSs40, EM6s137, EM8ws146 and MRw207 were moderate halophiles, with optimal growth at $10 \%$ salt, and a varying minimum requirement of $2 \%$ to $10 \%$ solar salt for growth. The STI histograms were right-skewed and showed an extreme halotolerance level of $20 \%$ to $25 \%$ and even to $30 \%$ solar salt.

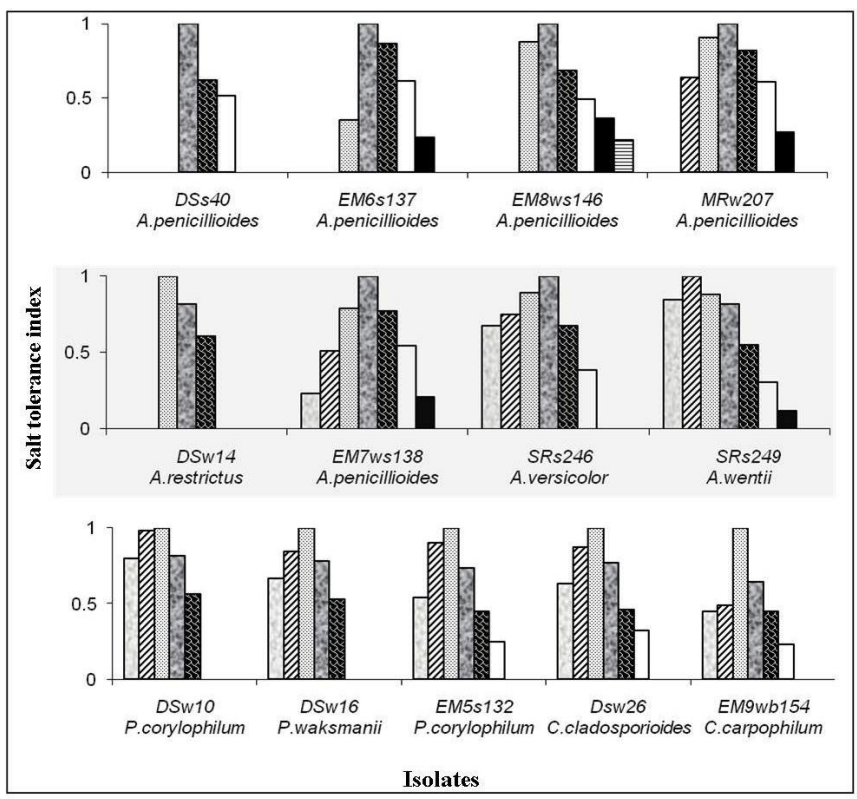

Fig. 1 Salt tolerance index of the halophilic isolates on CzA with solar salt: $0 \%$ ( $2 \%(\%), 5 \%(\%), 10 \%($ (W) $), 15 \%($ ) $, 20 \%(\quad), 25 \%($ a $)$ and $30 \%(\equiv)$
DSw14 Aspergillus restrictus was grouped under facultative halophiles, as it showed a delayed growth at $0-2 \%$ solar salt at 15 days incubation, which is not shown in the STI calculation. The facultative halophilic isolates of aspergilli (DSw14 Aspergillus restrictus, $\mathrm{EM} \mathrm{w}_{\mathrm{s}} 138$ A. penicillioides and SRs246 A. versicolor) penicilli (EM5s132 P. corylophilum and DSw16 P. waksmanii) and cladosporia (DSw26 C. cladosporioides and EM9 $\mathrm{w}_{\mathrm{b}} 154$ C. Carpophilum) were also all moderate halophiles, with an optimal growth in the presence of $5 \%$ or $10 \%$ salt. The facultative halophilic fungi (SRs249 Aspergillus wentii and DSw10 Penicillium corylophilum) were slight halophiles with a growth optimum around $2 \%$ solar salt. All the facultative halophilic isolates showed an extreme halotolerance level of $15 \%$ to $25 \%$ solar salt, with either a bell-shaped or right-skewed histogram.

The obligate halophiles exhibited an STI of $>0.5$ at salt concentrations of $10-20 \%$, and of $5-15 \%$ and $2-20 \%$ for the facultative halophilic $A$. restrictus and A. Penicillioides, respectively, while that of the other facultatively halophilic aspergilli was $\geq 0.5$ at salt concentrations of $0-15 \%$. The STI at these concentrations of solar salt, by the different genera was in the order of cladosporia $<$ penicilli $<$ aspergilli.

Significant difference $(\mathrm{P}<0.05)$ was obtained in the STI of each isolate at different salt concentrations, and the STI of isolates within the obligate aspergilli, within the facultative aspergilli and the cladosporia, as well as within all the isolates of Aspergillus; however, there was similarity $(\mathrm{P}>0.05)$ in the STI of isolates of penicilli.

Osmolytes: The osmolytes detected in the given isolates were of four to six carbon chain, comprising sugars sucrose and trehalose, and polyols such as the four carbon chain erythritol, the five carbon chain ribitol, arabitol, xylitol, and the six carbon chain inositol, mannitol, and dulcitol, along with glucose and/or its epimer galactose, in the obligate halophilic Aspergillus penicillioides (DSs40, EM7ws137, EM8ws146 and MRw207), facultative isolates aspergilli (DSw14 A. restrictus, EM7ws138 A. penicillioides, SRs246 A. versicolor and SRs249 A. wentii), penicilli (DSw10 $P$. corylophilum, EM5s132 P. corylophilum and DSw16 $P$. waksmanii) and cladosporia (DSw26 C. cladosporioides and EM9 $\mathrm{w}_{\mathrm{b}} 154$ C. carpophilum). There was a significant difference $(\mathrm{P}<0.5)$ in the relative concentrations of the osmolytes in each isolate (Fig. 2).

In the obligate halophilic aspergilli $A$. penicillioides, both glucose and galactose were identified in DSs40, EM7ws 137 and MRw207, while only galactose was detected in EM8ws146. The facultative halophilic aspergilli EM7ws138 A. penicillioides and SRs246 A. versicolor accumulated galactose, while glucose was found in SRs249 A. wentii. Glucose was also detected in all the penicilli, and to a lesser extent in cladosporia.

As shown in Fig. 3, the obligate aspergilli accumulated total of 4-14 mg sugars $\mathrm{g}^{-1}$ mycelia wet weight and 14-37 mg polyols $\mathrm{g}^{-1}$ mycelia wet weight. Between the different isolates there was a significant difference $(\mathrm{P}<0.5)$ in the total sugars and total polyols accumulated. Amongst the facultative halophiles, aspergilli accumulated total of 5-11 mg sugars $\mathrm{g}^{-1}$ 


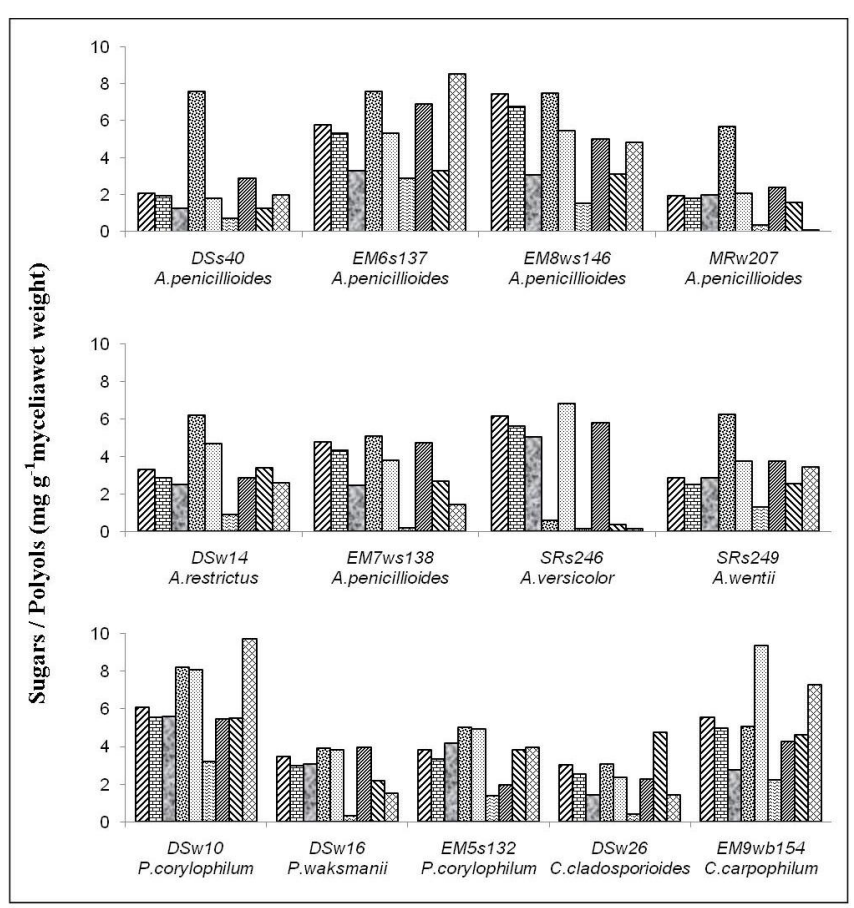

Fig. 2 Intracellular sugars and polyols in the halophilic isolates: Sucrose $(\%)$, trehalose

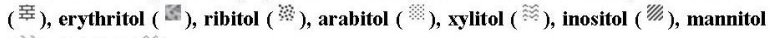
(N), dulcitol ( ).

mycelia wet weight and $18-23 \mathrm{mg}$ polyols $\mathrm{g}^{-1}$ mycelia wet weight, penicilli accumulated total of 6-11 $\mathrm{mg}$ sugars and total of 18-45 mg polyols $\mathrm{g}^{-1}$ mycelia wet weight, while the amount of total sugars and polyols accumulated in $C$. cladosporioides DSw26 was $5.5 \mathrm{mg}$ and $15.7 \mathrm{mg} \mathrm{g}^{-1}$ mycelial wet weight, respectively, and in C. carpophilum EM9wb154 it was $10.5 \mathrm{mg}$ and $35.35 \mathrm{mg} \mathrm{g}^{-1}$ mycelia wet weight, respectively. There was no significant difference $(\mathrm{P}>0.5)$ between the facultative aspergilla, however, a significant difference $(\mathrm{P}<0.5)$ was seen amongst isolates of Penicillium and of Cladosporium, in the total sugars and total polyols accumulated.

\section{DISCUSSION}

The salt tolerance index (STI) of the isolates displayed their euryhaline nature, able to adapt to a wide range of salt concentrations, the obligate halophiles however, showing a narrower tolerance range below the optimal salt concentration as seen from the right skewed histogram, compared to that of the facultative halophiles. It has also been shown earlier that conidia of the obligate halophiles could germinate in the absence of solar salt, but could not grow thereafter (Nazareth and Gonsalves, 2014b). The STI also indicated the degree of halophilicity, the obligate halophiles having a greater STI value at a higher salt range, as compared to that of the facultative halophiles. Amongst the facultative halophiles, A. restrictus and A. penicillioides, so classified because of their ability to grow in the absence of solar salt, had a greater STI value at a higher salt range than that of the other facultative halophiles of Aspergillus, Penicillium and Cladosporium, which grew more readily in the absence of solar salt, thus showing the stronger halophilic nature of these two isolates over that of the latter.

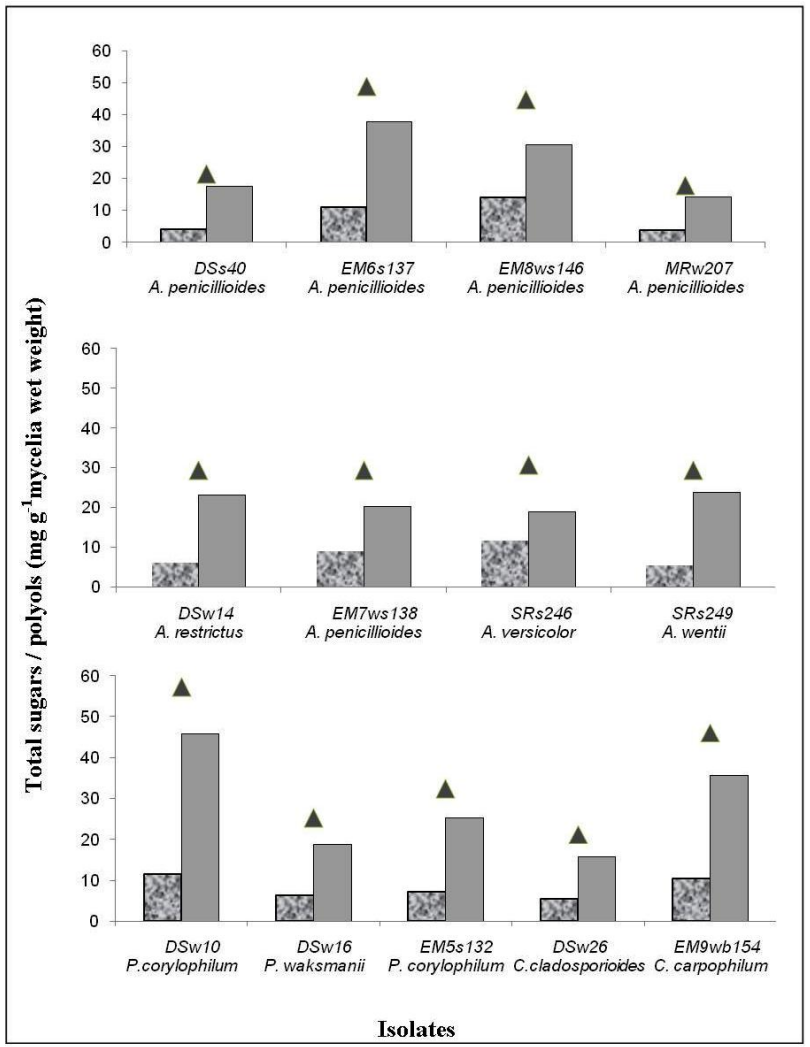

Fig. 3 Intracellular total sugars and total polyols in the halophilic isolates: sugars ( $)$, polyols ( $\square$ ), sugars + polyols $(\boldsymbol{\Delta})$

The detection of a consortium of various polar uncharged compatible solutes produced by the isolates in response to osmotic stress of sodium chloride, which comprised sugars sucrose and trehalose, and polyols such as erythritol, ribitol, arabitol, and mannitol, corroborated earlier records, but were detected in higher concentrations than reported earlier (Beever and Laracy, 1986; Kelavkar and Chhatpar, 1993; ElKady et al., 1995; Davis et al., 2000; Nesci et al., 2004; Ramirez et al., 2004; Roberts, 2005; Bois et al., 2006, Dijksterhuis and Vries, 2006; Kogej et al., 2007; Plemenitas et al., 2008; Kumar and Gummadi, 2009); in addition xylitol, inositol and dulcitol were also detected.

The production of high concentrations of compatible solutes would have contributed to the wide range of salt tolerance by the organisms, particularly of the facultative halophiles, as it is known that organisms employing the mechanism of osmolyte production as a means of osmoregulation, are more flexible, with a higher degree of adaptability than those employing the salt-in-cytoplasm mechanism, as the intracellular concentrations of organic osmolytes can be upor down- regulated according to the salinity of the medium (Kunte et al., 2002; Yancey, 2005).

The polyol osmolytes in the obligate halophiles were in greater concentration than the sugar osmolytes, and thus appear to have a significant role in the osmoadaptation mechanism of these fungi. The various polyol osmolytes produced by the isolates comprised 4 to 6 carbon chain with a corresponding increase in molecular weight (MW), namely, 
the 4 carbon chain erythritol with MW $122 \mathrm{gmol}^{-1}$, the 5 carbon moieties ribitol, arabitol, xylitol with MW152 $\mathrm{g} \mathrm{mol}^{-1}$ and the six carbon chain inositol, mannitol, and dulcitol, with MW182 $\mathrm{g} \mathrm{mol}^{-1}$. It could be expected that the increase in the polyol chain, with its corresponding higher molecular size, would have an increasing capacity for osmoregulation. It was also noted that the isolates produced a higher total concentration of 5 carbon and 6 carbon poyols than that of the 4 carbon erythritol, which would also have contributed to a more efficient osmoprotection. Shen et al., (1999) also reported that during salt stress, yeast cells had more of 6 carbon polyols than the 3 carbon glycerol. This is in accordance with the earlier records that polyols may be differentially active as compatible solutes, depending on their molecular weight, wherein larger molecular size polyols render higher osmotic pressures than smaller ones (Hallsworth and Magan, 1996; Davis et al., 2000).

A consortium of compatible solutes has been noted to be more efficient in increasing water pressure than a single compound, since individual compounds are differentially active as compatible solutes (Ramirez et al.,2004). A mixture also helps to avoid the toxicity associated with high concentration of a single osmolyte that can cause destabilization of proteins and interference in cellular processes such as protein - protein interaction and DNA protein interaction, and also prevents feedback mechanisms that can down regulate metabolic pathways in the presence of a high concentration of the product (Kogej et al., 2007; Singh et al., 2011). Moreover the varied osmolytes do not compete with each other (Holthauzen and Bolen, 2007) and may in fact act synergistically (Davis et al., 2000).

Among the sugars detected in the isolates, sucrose was dominant. It may be transported intracellularly from the medium, serving a transient role as a compatible solute (Beever and Laracy, 1986; Grant, 2004; Roberts, 2005), and serves as a good source for polyol production, eventually being replaced by other, more effective osmolytes. Reducing sugars, such as glucose and its epimer galactose, were also detected and may function as intermediates in the synthesis of osmolytes. Gaunt and Manners, (1973) reported that glucose serves for trehalose production. Roberts, (2005) reported that only a few carbohydrates are used for osmotic balance since those with reducing end are chemically reactive and can react with surface amino groups of proteins. Trehalose is used not only as a reserve carbohydrate but also as an osmoprotectant (Bois et al., 2006), serving to counteract dessication, and has been linked to longevity (Kuehn et al., 1998; Dijksterhuis and De Vries, 2006; Uyar et al., 2010), functioning also as a protective solute in stress conditions of high and low temperatures, as well as metal toxicity (Kuehn et al., 1998).

Among the polyols detected, mannitol, erythritol, and arabitol have been well documented in reports as cited above. However, inositol, a polar uncharged solute, which was one of the major polyols detected in both obligate as well as facultative halophilic isolates, has been only tentatively identified in small amounts as an osmolyte in Aspergillus nidulans (Beever and Laracy, 1986). This paper also reports the presence of xylitol and dulcitol amongst the osmolytes, hitherto unreported. Furthermore, a greater spectrum of osmolytes was detected in a given halophilic fungal isolate, than so far reported.

\section{CONCLUSIONS}

A commonality of the solutes was observed in both obligate and facultative halophiles, as well as in all three genera of the facultative halophiles, although they may have varied in relative concentrations, suggesting these may have a significant role in osmoadaptation in all halophilic filamentous fungi. The osmolytes did not appear to have a specific correlation to the type of saline econiche: thalassohaline or athalassohaline, hypersaline or polyhaline from which they had been isolated, or the obligate or facultative halophilic nature and degree of halophilicity or hatolerance of the isolate, nor to a specific genus or species. These results show that halophilic fungi can serve as valuable source of compatible solutes with a great potential for biotechnological applications.

\section{ACKNOWLEDGEMENT}

The authors gratefully acknowledge the laboratory facilities of Goa University, Goa and National Chemical Laboratory, Pune, during the course of this research.

\section{REFERENCES}

Beever, R.E. and Laracy, E.P. 1986. Osmotic adjustment in the filamentous fungus Aspergillus nidulans. $J$. Bacteriol.168:1358-1365.

Bois, G. et al. 2006. Growth, compatible solute and salt accumulation of five mycorrhizal fungal species grown over a range of $\mathrm{NaCl}$ concentrations. Mycorrhiza 16: 99-109.

Davis, D.J. 2000. Osmotic pressure of fungal compatible osmolytes. Mycol. Res. 104: 800-804.

DasSarma, S. and Arora, P. 2002. Halophiles. In: Encyclopedia of life sciences, vol 8. Nature Publishing Group, London, 458-466.

Dijksterhuis, J. and De Vries, R.P. 2006. Compatible solutes and fungal development. Biochem.J. 399 (2): e3-5.

El-Kady, I.A., Moubasher, M.H. and Mostafa, M.E. 1995. Accumulation of sugar alcohols by filamentous fungi. Folia Microbiol. 40: 481-486.

Gaunt, R.E. and Manners, J.G. 1973. The production of trehalose and polyols by Ustilago nuda in culture and their utilization in healthy and infected wheat plants. New Phytol. 72: 321-327.

Gonsalves, V., Nayak, S. and Nazareth, S. 2012. Halophilic fungi in a polyhaline estuarine habitat. J. YeastFungal Res. 3: 30-36.

Grant, W.D. 2004. Life at low water activity. Phil. Trans. R. Soc. Lond. 359 (1448): 1249-1267.

Hallsworth, J.E. and Magan, N. 1996. Culture age, temperature, and $\mathrm{pH}$ affect the polyol and trehalose contents of fungal propagules. Appl. Environ. Microb. 62:2435-2442. 
Holthauzen, L.M.F. and Bolen, D.W. 2007. Mixed osmolytes: The degree to which one osmolyte affects the protein stabilizing ability of another. Protein Sci. 16(2): 293-298.

Kelavkar, U.P. and Chhatpar, H.S. 1993. Polyol concentrations in Aspergillus repens grown under salt stress. World J. Microb. Biot. 9: 579-682.

Kogej, T. et al.2006. Mycosporines in extremophilic fungi novel complementary osmolytes? Environ. Chem. 3: 105110.

Kogej, T., et al. 2007. Osmotic adaptation of the halophilic fungus Hortaea werneckii: role of osmolytes and melanization. Microbiol. 153: 4261-4273.

Kuehn, K.A., Churchill, P.F. and Suberkropp, K. 1998. Osmoregulatory responses of fungi inhabiting standing litter of the freshwater emergent macrophyte Juncus effuses. Appl Environ. Microb. 64: 607-612.

Kumar, S., and Gummadi, S.N. 2009. Osmotic adaptation in halotolerant yeast, Debaryomyces nepalensis NCYC 3413: role of osmolytes and cation transport. Extremophiles. 13: 793-805.

Kunte, J.H., Truper, H.G. and Stan-Lotter, S. 2002. Halophilic microorganisms. In: Astrobiology: the Quest for the conditions of life. (Eds.: Horneck, G. and Baumstark, C.) Springer, Berlin, pp 185-199 pp.

Nayak, S., Gonsalves, V. and Nazareth, S. 2012. Isolation and salt tolerance of halophilic fungi from mangroves and solar salterns in Goa India. Indian J. Geo-Mar. Sci. 41: 164-172.

Nazareth, S. and Gonsalves, V. 2014a.Aspergillus penicillioides - a true halophile existing in hypersaline and polyhaline econiches. Ann. Microbiol. 64: 397-402.

Nazareth, S. and Gonsalves, V. 2014b. Halophilic Aspergillus penicillioides from athalassohaline, thalassohaline, and polyhaline environments. Front. Microbiol. 5: 1-5.

Nazareth, S., Gonsalves, V. and Nayak, S. 2012. A first record of obligate halophilic aspergilli from the Dead Sea. Indian J. Microbiol. 52: 22-27.
Nazareth, S.W. 2014. The world of halophilic fungi. Kavaka 42: 131-144.

Nesci, A., Etcheverry, M. and Magan, N. 2004. Osmotic and matric potential effects on growth, sugar alcohol and sugar accumulation by Aspergillus section Flavi strains from Argentina. J. Appl. Microbiol. 96: 965-972.

Plemenitas, A., Vaupotic, T., Lenassi, M., Kojej, T. and Gunde-Cimerman, N. 2008. Adaptation of extremely halotolerant black yeast Hortaea werneckii to increased osmolarity: a molecular perspective at a glance. Stud. Mycol. 61:67-75.

Ramirez, M.L., Chulze, S.N. and Magan, N. 2004. Impact of osmotic and matric water stress on germination, growth, mycelia water potentials and endogenous accumulation of sugars and sugar alcohols in Fusarium graminearum. Mycologia 96: 470-478.

Roberts, M.F. 2005. Organic compatible solutes of halotolerant and halophilic microorganisms.Saline Systems 1: 5.

Shen, B. et al. 1999. Roles of sugar alcohols in osmotic stress adaptation. Replacement of glycerol by mannitol and sorbitol in Yeast. Plant Physiol.121:45-52

Singh, L.R. et al. 2011. Protein and DNA destabilization by osmolytes: The other side of the coin. Life Sci. 88: 117125.

Uyar, E.O., Hamamci, H. and Türkel, S. 2010. Effect of different stresses on trehalose levels in Rhizopus oryzae. J. Basic Microb. 50: 368-372.

Yancey, P.H. 2005. Organic osmolytes as compatible, metabolic and counteracting cytoprotectant in high osmolarity and other stresses. J. Exp.Biol. 208: 28192830. 